\title{
Effect of Irrigation Levels and Cropping Systems on Quality Parameters and Economics of Pigeonpea
}

\author{
Megharani", Pandit, S. Rathod", B. M. Dodamani, Devappagouda H. Patil and R. P. Patil \\ Department of Agronomy, Zonal Agricultural Research Station, Aland Road, \\ Kalaburgi-585101, Karnataka, India \\ *Corresponding author
}

\section{A B S T R A C T}

\section{Keywords}

CPE, Irrigation scheduling, Cropping systems, Pigeonpea, WUE

\section{Article Info}

Accepted:

15 January 2020 Available Online: 10 February 2020
A field experiment was conducted to study "Effect of irrigation levels and cropping systems on quality parameters and economics of pigeonpe" at Zonal Agricultural Research Station, Kalaburagi, during kharif 2018-19. The experiment was laid out in split plot design with three main factors of irrigation levels and four sub factors of cropping systems, replicated thrice revealed that significantly higher nitrogen uptake $\left(161.04 \mathrm{~kg} \mathrm{ha}{ }^{-1}\right)$ phosphorus uptake $\left(28.26 \mathrm{~kg} \mathrm{ha}^{-1}\right)$, potassium uptake $\left(85.29 \mathrm{~kg} \mathrm{ha}^{-1}\right)$, protein yield $(590.73$ $\mathrm{kg} \mathrm{ha}^{-1}$ ), grain yield $\left(1575 \mathrm{~kg} \mathrm{ha}^{-1}\right.$ ) and economics viz., gross returns (Rs. 2,26,245 ha-1), net returns (Rs.173435 ha ${ }^{-1}$ ) and $\mathrm{BC}$ ratio (4.29) with $75 \% \mathrm{CPE}$ ratio compared to50\% and $100 \%$ CPE but water use efficiency $\left(5.59 \mathrm{Kg} \mathrm{ha}^{-1} \mathrm{~mm}^{-1}\right)$ of pigenopea highest in $50 \%$ $\mathrm{CPE}$ compared to $75 \% \mathrm{CPE}$ and $100 \% \mathrm{CPE}$. Among the cropping system, sole pigeonpea recorded significantly higher nitrogen uptake $\left(176.72 \mathrm{~kg} \mathrm{ha}^{-1}\right)$, phosphorus uptake (33.56 $\left.\mathrm{kg} \mathrm{ha}^{-1}\right)$, potassium uptake $\left(89.70 \mathrm{~kg} \mathrm{ha}^{-1}\right)$, protein yield $\left(635.37 \mathrm{~kg} \mathrm{ha}^{-1}\right)$, WUE (4.66 Kg $\left.\mathrm{ha}^{-1} \mathrm{~mm}^{-1}\right)$ and grain yield $\left(1732 \mathrm{~kg} \mathrm{ha}^{-1}\right)$ as compared to intercropped pigeonpea. While, pigeonpea + greengram (1:3) intercropping system recorded significantly higher gross returns (Rs. $252400 \mathrm{ha}^{-1}$ ), net returns (Rs. $196590 \mathrm{ha}^{-1}$ ) and $\mathrm{BC}$ ratio (4.52) as compared to sole pigeonpea.

\section{Introduction}

Pulses are the important sources of proteins, vitamins and minerals and are popularly known as "Poor man's meat" and "rich man's vegetable", as they contribute significantly to the nutritional security of the country. The importance of pulses is much more in country like India, where majority of the people are vegetarian. Unfortunately, the pulse production in the country has not been able to keep pace with the increasing population. The demand of pulses is increasing at a faster rate to meet the minimum protein requirements of an increasing world population. In India, pigeonpea is cultivated in an area of 4.78 million hectares with production of 4.25 million tones and productivity of $889 \mathrm{~kg}$ per hectare (Anon., 2018) and pigeonpea requires about $63.3 \mathrm{~kg} \mathrm{~N}, 15.8 \mathrm{~kg} \mathrm{P}_{2} \mathrm{O}_{5}$ and $49.8 \mathrm{~kg}$ $\mathrm{K}_{2} \mathrm{O}$ per hectare to produce 1 tonne of pigeonpea grains (Patil and Padmani, 2007). 
Nutrient uptake plays a significant role in deciding the pigeonpea yield and knowing the level of nutrient accumulation in the crop helps in the formulating the efficient fertilization schedule, as most of the applied nutrients are lost through leaching, volatilization, denitrification and chemical fixation soil (Chandrasekhar et al., 2015)

Increasing demand for irrigation water coupled with depleting ground water sources calls for efficient use of water. Therefore, there is a need for efficient irrigation methods like micro irrigation. The present scenario of flood irrigation should be replaced by more efficient and controlled irrigation systems. Therefore, use of modern irrigation systems like drip irrigation provides better crop growth and greater yields, due to efficient use of water and nutrients. Among the various techniques advocated for economizing water use, scheduling of irrigation based on CPE ratio is considered to be the most effective and important. Water allocation and scheduling of irrigation based on the data of the pan evaporation are likely to result not only in better pigeonpea production but also increase water use efficiency.

\section{Materials and Methods}

A field experiment was conducted during the kharif 2018-19 at Zonal Agriculture Research Station, Kalaburgi, is situated at North Eastern Dry Zone of Karnataka at a latitude of $17^{\circ} 34^{\prime}$ North, longitude of $76^{\circ} 79^{\prime}$ East and an altitude of 478 meters above mean sea level (MSL). The experiment was laid out in split plot design with three main factors of irrigation levels $\mathrm{I}_{1}: 50 \% \mathrm{CPE}, \mathrm{I}_{2}: 75 \% \mathrm{CPE}$ and $\mathrm{I}_{3}: 100 \% \mathrm{CPE}$ and four sub factors of cropping systems $\mathrm{C}_{1}$ : Sole pigeon pea, $\mathrm{C}_{2}$ : Pigeonpea + Greengram, $\mathrm{C}_{3}$ : Pigeonpea + Blackgram and $\mathrm{C}_{4}$ : Pigeonpea + Soybean, replicated thrice and Rainfed pigeonpea outside the control. Recommended dose of fertilizer for pigeonpea (25:50:0 $\mathrm{kg} \mathrm{N}$ : $\mathrm{P}_{2} \mathrm{O}_{5}$ : $\mathrm{K}_{2} \mathrm{O}$ ) were applied at the time of sowing. Nitrogen, phosphorous and potassium were applied in the form of diammonium phosphate (DAP) and Farm yard manure (FYM) @ $6 \mathrm{t} \mathrm{ha}^{-1}$ was incorporated into soil two weeks before sowing. Later on water soluble fertilizers were applied through drip irrigation viz., $4 \mathrm{~kg}$ of 19:19:19 (N: P: K) and $8.5 \mathrm{~kg}$ of MAP (12:61:0) throughout the growing period of crop. The sowing was done on $13^{\text {th }}$ June, 2018. The total rainfall received during the cropping season $549.80 \mathrm{~mm}$.

The soil of the experimental site was black clay, slightly alkaline (8.20) with an electrical conductivity of $0.23 \mathrm{dS} \mathrm{m} \mathrm{m}^{-1}$. The soil organic carbon content was low $(0.52 \%)$. The soil was low in available nitrogen (235 kg ha-1), medium in available phosphorus $\left(32 \mathrm{~kg} \mathrm{ha}^{-1}\right)$ and high in available potassium (460 kg ha ${ }^{-1}$ ) respectively. Soil application of fertilizers at 30 DAS and foliar application of fertilizers at $50 \%$ flowering stage.

\section{Results and Discussion}

\section{Nutrients uptake}

The nitrogen, phosphorus and potassium uptake by pigeonpea as influenced by irrigation scheduling through drip and cropping systems are presented in Table 1.

Among the different irrigation levels, scheduling of irrigation at $75 \% \mathrm{CPE}$ recorded significantly higher nitrogen (161.04 $\mathrm{kg} \mathrm{ha}^{-1}$ ), phosphorus (28.26 kg ha-1) and potassium $\left(85.29 \mathrm{~kg} \mathrm{ha}^{-1}\right)$ uptakes in pigeonpea and significantly lower nitrogen (116.70 kg ha ${ }^{-1}$ ), phosphorus (20.87 $\mathrm{kg} \mathrm{ha}^{-1}$ ) and potassium $\left(49.87 \mathrm{~kg} \mathrm{ha}^{-1}\right)$ uptakes was recorded in $50 \%$ CPE. Scheduling of irrigation at $75 \% \mathrm{CPE}$ recorded significantly higher nitrogen, phosphorus and potassium uptake, this was mainly due to higher dry matter production 
and yield and also due to maintenance of optimum moisture condition which helped for better root penetration and proliferation leading to higher nutrient uptake involving diffusion, mass flow and root interception. Soil with optimum water content helps for better solubility of nutrients than limited water status as well as reduces the chances of leaching major part of nutrient due to excess water leads to higher uptake (Shiragapure and Fathima, 2018). Singh et al., (2018) reported that drip irrigation at $100 \% \mathrm{WRc}$ registered significantly higher nitrogen, phosphorus and potassium uptake compared to other level of irrigation. These results are in conformity with Al-Kaisi and Yin (2003) in maize and Hebbar et al., (2004) in tomato.

Among the different cropping systems, sole crop of pigeonpea recorded significantly higher nitrogen uptake (176.72 $\mathrm{kg} \mathrm{ha}^{-1}$ ) phosphorus uptake $\left(33.56 \mathrm{~kg} \mathrm{ha}^{-1}\right)$ and potassium uptake $\left(89.70 \mathrm{~kg} \mathrm{ha}^{-1}\right)$ than intercropped pigeonpea. Between the intercropping system, pigeonpea + soybean in 1:3 row proportions recorded significantly higher nutrient uptake as compared to pigeonpea + greengram (1:3) and pigeonpea + blackgram (1:3) intercropping systems and significantly lower nutrient uptake was recorded in pigeonpea + greengram (1:3) intercropping system. Sole pigeonpea recorded significantly higher uptake of $\mathrm{N}, \mathrm{P}$ and $\mathrm{K}$ mainly due to higher seed yield along with higher nutrient contents present in seed. Nutrient uptake is mainly governed by nutrient content and yield. Significantly higher grain yield, yield components and total dry matter production per plant are mainly contributed for higher total uptake of nutrient by pigeonpea.

Interaction effect between scheduling of irrigation and cropping system on nutrient uptake was found significant. Scheduling of irrigation at $75 \% \mathrm{CPE}$ with sole pigeonpea recorded significantly higher nitrogen uptake (190.04 kg ha $\left.{ }^{-1}\right)$, phosphorous uptake (34.02 $\mathrm{kg} \mathrm{ha}^{-1}$ ) and potassium uptake (92.46 kg ha-1) as compared to rest of the treatment combinations. However, significantly lower nitrogen uptake $\left(69.00 \mathrm{~kg} \mathrm{ha}^{-1}\right)$, phosphorous uptake (16.10 kg ha $\left.{ }^{-1}\right)$ and potassium uptake $\left(34.92 \mathrm{~kg} \mathrm{ha}^{-1}\right)$ was registered in $50 \% \mathrm{CPE}$ with pigeonpea + greengram (1:3) intercropping system.

The rainfed zpigeonpea recorded significantly lower nitrogen uptake $\left(65.60 \mathrm{~kg} \mathrm{ha}^{-1}\right)$, phosphorus uptake $\left(14.56 \mathrm{~kg} \mathrm{ha}^{-1}\right)$ and potassium uptake $\left(31.35 \mathrm{~kg} \mathrm{ha}^{-1}\right)$.

\section{Protein yield}

Protein yield was significantly higher under scheduling of irrigation at $75 \%$ CPE (590.73 $\mathrm{kg} \mathrm{ha}^{-1}$ ) as compared to $50 \% \mathrm{CPE}$ and $100 \%$ CPE levels. But, it was on par with scheduling of irrigation at $100 \% \mathrm{CPE}$ $\left(558.58 \mathrm{~kg} \mathrm{ha}^{-1}\right)$ and significantly lower protein yield was recorded in $50 \% \mathrm{CPE}$ (510.16 $\mathrm{kg} \mathrm{ha}^{-1}$ ) (Table 2). This might be due to increase in root proliferation which facilitated the higher uptake of nitrogen from the soil resulting into higher protein content and higher protein yield. Similar results were found by Kumbhar et al., (2015).

Different cropping systems significantly influenced the protein yield. Among the intercropping system, significantly higher protein yield was recorded in sole pigeonpea (635.37 $\mathrm{kg} \mathrm{ha}{ }^{-1}$ ) as compared to the intercropped pigeonpea. Between the intercropping systems, pigeonpea intercropped with soybean in 1:3 row proportions $\left(535.48 \mathrm{~kg} \mathrm{ha} \mathrm{ha}^{-1}\right.$ recorded significantly higher protein yield as compared to pigeonpea + greengram (1:3) and pigeonpea + blackgram (1:3) intercropping systems and are on par with each other (Table 2). This was probably due to comparatively 
greater availability of nutrients to the sole pigeonpea as compared to intercropping where it was shared by both the crops thereby increases protein yield. The results are in close conformity with the findings of Abraham et al., (2010).

The interaction effect due to scheduling of irrigation and cropping systems on protein yield of pigeonpea was found significant. Scheduling of irrigation at $75 \% \mathrm{CPE}+$ sole pigeonpea $\left(685.61 \mathrm{~kg} \quad \mathrm{ha}^{-1}\right)$ recorded significantly higher protein yield as compared to rest of the treatment combinations (Table 2). But it was found on par with $100 \% \mathrm{CPE}$ with sole pigeonpea (639.63 $\left.\mathrm{kg} \mathrm{ha}^{-1}\right)$. However, significantly lower protein yield was recorded in $50 \% \mathrm{CPE}$ with pigeonpea + greengram (1:3) intercropping system (476.41 $\mathrm{kg} \mathrm{ha}^{-1}$ ).

The rainfed pigeonpea recorded significantly lower protein yield $\left(455.60 \mathrm{~kg} \mathrm{ha}^{-1}\right)$.

\section{Water use efficiency}

Scheduling of irrigation at $50 \%$ CPE recorded significantly higher water use efficiency of pigeonpea $\left(5.59 \mathrm{~kg}\right.$ ha- $\mathrm{mm}^{-1}$ ) when compared to $75 \% \mathrm{CPE}$ and $100 \%$ CPE. Significantly lower water use efficiency was registered in $100 \%$ CPE $(3.03 \mathrm{~kg}$ ha$\mathrm{mm}^{-1}$ ) (Table 2).

The higher water use efficiency might be due to very less amount of irrigation water applied at $50 \%$ CPE $(407.4 \mathrm{~mm})$ compared to irrigation at $75 \% \mathrm{CPE}(611.3 \mathrm{~mm})$ and 100 $\%$ CPE $(814.3 \mathrm{~mm})$. Although irrigation at 75 $\%$ CPE recorded more seed yield it could not gave more irrigation water use efficiency due to more water applied and comparatively less increase in seed yield compared to irrigation at $50 \%$ CPE. Thus higher seed yield was masked by higher amount of water applied under irrigation at $75 \% \mathrm{CPE}$ and $100 \% \mathrm{CPE}$, respectively, for improving water use efficiency in case of pigeonpea. The results are in arrangement with Jadhav et al., (2018), Muniyappa et al., (2017) and Ranjitha et al., (2018).

Water use efficiency of pigeonpea differed significantly due to different cropping systems. Significantly higher water use efficiency was recorded in sole pigeonpea (4.66 kg ha-mm $\mathrm{mm}^{-1}$ as compared to intercropped pigeonpea.

Among the cropping systems, pigeonpea intercropped with soybean in 1:3 row proportions recorded significantly higher

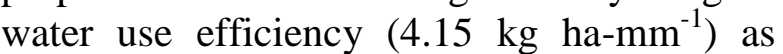
compared to pigeonpea + greengram $(1: 3)$ and pigeonpea + blackgram (1:3) intercropping systems. However, significantly lower water use efficiency was recorded in pigeonpea + greengram (1:3) intercropping system (4.04 $\mathrm{kg} \mathrm{ha} \mathrm{mm}^{-1}$ ) (Table 2). This might be due to higher water requirement of both the component crops under intercropping system, but sole pigeonpea required less water for their growth and development compared to intercrops (greengram, blackgram and soybean) hence sole pigeonpea recorded higher water use efficiency.

The interaction effect between scheduling of irrigation and cropping systems on water use efficiency of pigeonpea was found significant. Scheduling of irrigation at $50 \% \mathrm{CPE}$ with sole pigeonpea recorded significantly higher water use efficiency $\left(6.14 \mathrm{~kg}\right.$ ha- $\left.\mathrm{mm}^{-1}\right)$ when compared to rest of the treatment combinations, but it was on par with scheduling of irrigation at $50 \% \mathrm{CPE}$ with pigeonpea + blackgram $\left(5.44 \mathrm{~kg}\right.$ ha- $\left.\mathrm{mm}^{-1}\right)$ and $50 \%$ CPE with pigeonpea + soybean $\left(5.44 \mathrm{~kg} \mathrm{ha}-\mathrm{mm}^{-1}\right)$ intercropping system (1:3). Significantly lower water use efficiency was registered in $100 \% \mathrm{CPE}$ with sole pigeonpea $\left(2.88 \mathrm{~kg} \mathrm{ha}^{-\mathrm{mm}^{-1}}\right.$ ) (Table 2). 


\section{Grain yield}

In the present investigation, significant difference was noticed in yield of pigeonpea as influenced by irrigation scheduling and cropping system was presented in table 3 . Among the irrigation levels, scheduling of irrigation at $75 \% \mathrm{CPE}$ recorded significantly higher seed yield of pigeonpea $\left(2524 \mathrm{~kg} \mathrm{ha}^{-1}\right)$ as compared to $50 \%$ and $100 \% \mathrm{CPE}$, but it was on par with scheduling of irrigation at $100 \%$ CPE $\left(2462 \mathrm{~kg} \mathrm{ha}^{-1}\right)$ and significantly lower seed yield was recorded in $50 \%$ CPE $\left(2276 \mathrm{~kg} \mathrm{ha}^{-1}\right)$. This may be due to continuous and controlled supply of irrigation water throughout the crop growth period. The adequate supply of sufficient moisture for prolonged period also exploits the fixed nutrients of soil in available form and regulates its supply to the crop through mineralization and prevents from leaching and other losses and thereby improve the yield (Kiran, 2014). These results are in close agreement with the findings obtained by Thanki and Solanki (2010) and Mansur et al., (2010).

Among the cropping systems, sole pigeonpea recorded significantly higher seed yield (2656 $\mathrm{kg} \mathrm{ha}{ }^{-1}$ ) as compared to intercropped pigeonpea. Between the intercropping systems, pigeonpea + soybean in 1:3 row proportions recorded significantly higher seed yield $\left(2366 \mathrm{~kg} \mathrm{ha}^{-1}\right)$ as compared to pigeonpea + greengram (1:3) and pigeonpea + blackgram (1:3) intercropping systems and are on par with each other.

The enhancement in yield may be due to no competition of main crop with intercrop. The results obtained from the present study contradict the findings by Ansari et al., (2012) who reported higher pigeonpea yield $(1.52 \mathrm{t}$ $\left.\mathrm{ha}^{-1}\right)$ under sole than intercrop plot $(0.61 \mathrm{t}$ $\left.\mathrm{ha}^{-1}\right)$.
Interaction effect between scheduling of irrigation and cropping system was found significant. Scheduling of irrigation at $75 \%$ CPE with sole crop of pigeonpea (2750 $\mathrm{kg} \mathrm{ha}^{-1}$ ) recorded significantly higher seed yield as compared to rest of the treatment combinations. But, it was on par with the treatments $100 \% \mathrm{CPE}$ with sole pigeonpea $\left(2718 \mathrm{~kg} \mathrm{ha}^{-1}\right), 50 \%$ CPE with sole pigeonpea $\left(2501 \mathrm{~kg} \mathrm{ha}^{-1}\right)$ and $75 \%$ CPE with pigeonpea + soybean $\left(2498 \mathrm{~kg} \mathrm{ha}^{-1}\right)$ intercropping system. However, significantly lower seed yield was recorded in 50\% CPE with pigeonpea + greengram (1:3) intercropping system $\left(2171 \mathrm{~kg} \mathrm{ha}^{-1}\right)$. Mahalakshmi et al., (2011) in pigeonpea and Muniyappa et al., (2017) in chickpea also reported the similar findings.

The rainfed pigeonpea recorded significantly lower seed yield $\left(1080 \mathrm{~kg} \mathrm{ha}^{-1}\right)$.

\section{Economics}

The data pertaining to gross returns, net returns and $\mathrm{BC}$ ratio as influenced by irrigation scheduling through drip and cropping systems are presented in Table 3. Scheduling of irrigation at $75 \%$ CPE recorded significantly higher gross returns (Rs. 2,26,245 ha ${ }^{-1}$ ), net returns (Rs. 1,73,435 $\mathrm{ha}^{-1}$ ) and BC ratio (4.29) as compared to $50 \%$ $\mathrm{CPE}$ and $100 \% \mathrm{CPE}$ and significantly lower gross returns was registered in $50 \%$ CPE (Rs. 1,96,020 ha' ${ }^{-1}$ ), net returns (Rs. 1,43,710 ha ${ }^{-1}$ ) and $\mathrm{BC}$ ratio (3.77). Jadhav et al., (2018) reported that irrigation at $75 \%$ Epan through drip recorded significantly higher gross returns (Rs. 1,33,683 ha ${ }^{-1}$ ), net returns (Rs. 96,995 $\mathrm{ha}^{-1}$ ) and BC ratio (3.64) followed by irrigation at $100 \%$ Epan through drip, irrigation at $50 \%$ Epan through drip and irrigation at $50 \%$ flowering through furrows, respectively. 
Table.1 Nutrient uptake of pigeonpea as influenced by irrigation levels and cropping systems under drip irrigation

\begin{tabular}{|c|c|c|c|}
\hline \multirow[t]{2}{*}{ Treatments } & \multicolumn{3}{|c|}{ Nutrient uptake } \\
\hline & $\begin{array}{c}\text { Nitrogen } \\
\left(\mathrm{kg} \mathrm{ha}^{-1}\right)\end{array}$ & $\begin{array}{l}\text { Phosphorus } \\
{\left(\mathrm{kg} \mathrm{ha}^{-1}\right)}\end{array}$ & $\begin{array}{c}\text { Potassium } \\
\left(\mathrm{kg} \mathrm{ha}^{-1}\right)\end{array}$ \\
\hline \multicolumn{4}{|l|}{ Main plots: Irrigation levels (I) } \\
\hline $50 \% \operatorname{CPE}\left(I_{1}\right)$ & 116.70 & 20.87 & 49.87 \\
\hline $75 \%$ CPE $\left(\mathrm{I}_{2}\right)$ & 161.04 & 28.26 & 85.29 \\
\hline $100 \% \mathrm{CPE}\left(\mathrm{I}_{3}\right)$ & 141.72 & 23.30 & 58.93 \\
\hline S.Em \pm & 3.70 & 0.74 & 2.28 \\
\hline C.D. at $5 \%$ & 14.52 & 2.89 & 8.94 \\
\hline \multicolumn{4}{|l|}{ Sub plot: Cropping systems (C) } \\
\hline Sole pigeonpea $\left(\mathbf{C}_{1}\right)$ & 176.72 & 33.56 & 89.70 \\
\hline Pigeonpea + Greengram $\left(\mathrm{C}_{2}\right)$ & 111.30 & 19.00 & 53.29 \\
\hline Pigeonpea + Blackgram $\left(\mathrm{C}_{3}\right)$ & 128.80 & 20.68 & 55.68 \\
\hline Pigeonpea + Soybean $\left(\mathbf{C}_{4}\right)$ & 142.30 & 23.32 & 60.12 \\
\hline S.Em \pm & 6.77 & 0.96 & 3.82 \\
\hline C.D. at $5 \%$ & 20.12 & 2.86 & 11.34 \\
\hline \multicolumn{4}{|l|}{ Interaction effects $(I \times C)$} \\
\hline $50 \% \mathrm{CPE} \times$ Sole pigeonpea $\left(\mathrm{I}_{1} \times \mathrm{C}_{1}\right)$ & 159.53 & 33.04 & 86.31 \\
\hline $50 \%$ CPE $\times$ Pigeonpea + Greengram $\left(I_{1} \times C_{2}\right)$ & 69.00 & 16.10 & 34.92 \\
\hline $50 \%$ CPE $\times$ Pigeonpea + Blackgram $\left(I_{1} \times C_{3}\right)$ & 110.72 & 16.35 & 36.90 \\
\hline $50 \%$ CPE $\times$ Pigeonpea + Soybean $\left(I_{1} \times C_{4}\right)$ & 127.04 & 17.98 & 41.35 \\
\hline $75 \%$ CPE $\times$ Sole pigeonpea $\left(I_{2} \times C_{1}\right)$ & 190.04 & 34.02 & 92.46 \\
\hline $75 \%$ CPE $\times$ Pigeonpea + Greengram $\left(I_{2} \times C_{2}\right)$ & 141.03 & 22.35 & 79.68 \\
\hline $75 \%$ CPE $\times$ Pigeonpea + Blackgram $\left(I_{2} \times C_{3}\right)$ & 145.60 & 26.35 & 80.45 \\
\hline $75 \%$ CPE $\times$ Pigeonpea + Soybean $\left(I_{2} \times C_{4}\right)$ & 167.50 & 30.34 & 88.57 \\
\hline $100 \%$ CPE $\times$ Sole pigeonpea $\left(I_{3} \times C_{1}\right)$ & 180.60 & 33.62 & 90.34 \\
\hline $100 \%$ CPE $\times$ Pigeonpea + Greengram $\left(I_{3} \times C_{2}\right)$ & 123.86 & 18.56 & 45.26 \\
\hline $100 \%$ CPE $\times$ Pigeonpea + Blackgram $\left(\mathrm{I}_{3} \times \mathrm{C}_{3}\right)$ & 130.07 & 19.35 & 49.68 \\
\hline $100 \%$ CPE $\times$ Pigeonpea + Soybean $\left(I_{3} \times C_{4}\right)$ & 132.35 & 21.65 & 50.43 \\
\hline S.Em \pm & 10.81 & 1.62 & 6.16 \\
\hline C.D. at $5 \%$ & 32.12 & 4.82 & 18.31 \\
\hline Rainfed pigeonpea & 65.6 & 14.56 & 31.35 \\
\hline S.Em \pm & 10.68 & 1.61 & 6.11 \\
\hline C.D. at $5 \%$ & 31.17 & 4.71 & 17.83 \\
\hline
\end{tabular}


Table.2 Protein content (\%), protein yield $\left(\mathrm{kg} \mathrm{ha}^{-1}\right)$ and water use efficiency $\left(\mathrm{Kg} \mathrm{ha}^{-1} \mathrm{~mm}^{-1}\right)$ of pigeonpea as influenced by irrigation levels and cropping systems under drip irrigation

\begin{tabular}{|c|c|c|c|}
\hline Treatments & $\begin{array}{c}\text { Protein } \\
\text { content } \\
(\%)\end{array}$ & $\begin{array}{l}\text { protein yield } \\
\left(\mathrm{kg} \mathrm{ha}^{-1}\right)\end{array}$ & $\begin{array}{c}\text { Water use } \\
\text { efficiency } \\
\left(\mathrm{Kg} \mathrm{ha}^{-1} \mathrm{~mm}^{-1}\right)\end{array}$ \\
\hline \multicolumn{4}{|l|}{ Main plots: Irrigation levels (I) } \\
\hline $50 \%$ CPE $\left(\mathbf{I}_{1}\right)$ & 22.32 & 510.16 & 5.59 \\
\hline $75 \%$ CPE $\left(I_{2}\right)$ & 23.34 & 590.73 & 4.13 \\
\hline $100 \%$ CPE $\left(\mathbf{I}_{3}\right)$ & 22.61 & 558.58 & 3.03 \\
\hline S.Em \pm & 0.65 & 16.02 & 0.11 \\
\hline C.D. at $5 \%$ & NS & 62.89 & 0.43 \\
\hline \multicolumn{4}{|l|}{ Sub plot: Cropping systems (C) } \\
\hline Sole pigeonpea $\left(C_{1}\right)$ & 23.83 & 635.37 & 4.66 \\
\hline Pigeonpea + Greengram $\left(C_{2}\right)$ & 22.27 & 514.27 & 4.04 \\
\hline Pigeonpea + Blackgram $\left(\mathbf{C}_{3}\right)$ & 22.37 & 527.50 & 4.14 \\
\hline Pigeonpea + Soybean $\left(\mathbf{C}_{4}\right)$ & 22.55 & 535.48 & 4.15 \\
\hline S.Em \pm & 0.69 & 20.33 & 0.15 \\
\hline C.D. at $5 \%$ & NS & 60.40 & 0.45 \\
\hline \multicolumn{4}{|l|}{ Interaction effects $(I \times C)$} \\
\hline $50 \%$ CPE $\times$ Sole pigeonpea $\left(I_{1} \times C_{1}\right)$ & 23.19 & 580.88 & 6.14 \\
\hline $50 \%$ CPE $\times$ Pigeonpea + Greengram $\left(I_{1} \times C_{2}\right)$ & 21.94 & 476.41 & 5.33 \\
\hline $50 \%$ CPE $\times$ Pigeonpea + Blackgram $\left(I_{1} \times C_{3}\right)$ & 22.00 & 487.45 & 5.44 \\
\hline $50 \%$ CPE $\times$ Pigeonpea + Soybean $\left(I_{1} \times C_{4}\right)$ & 22.15 & 495.88 & 5.44 \\
\hline $75 \% \mathrm{CPE} \times$ Sole pigeonpea $\left(\mathrm{I}_{2} \times \mathrm{C}_{1}\right)$ & 24.88 & 685.61 & 4.50 \\
\hline $75 \%$ CPE $\times$ Pigeonpea + Greengram $\left(I_{2} \times C_{2}\right)$ & 22.63 & 543.89 & 3.92 \\
\hline $75 \%$ CPE $\times$ Pigeonpea + Blackgram $\left(I_{2} \times C_{3}\right)$ & 22.81 & 556.76 & 4.01 \\
\hline $75 \%$ CPE $\times$ Pigeonpea + Soybean $\left(I_{2} \times C_{4}\right)$ & 23.06 & 576.65 & 4.09 \\
\hline $100 \% \mathrm{CPE} \times$ Sole pigeonpea $\left(\mathrm{I}_{3} \times \mathrm{C}_{1}\right)$ & 23.44 & 639.63 & 3.34 \\
\hline $100 \%$ CPE $\times$ Pigeonpea + Greengram $\left(I_{3} \times C_{2}\right)$ & 22.25 & 522.52 & 2.88 \\
\hline $100 \%$ CPE $\times$ Pigeonpea + Blackgram $\left(I_{3} \times C_{3}\right)$ & 22.31 & 538.28 & 2.96 \\
\hline $100 \%$ CPE $\times$ Pigeonpea + Soybean $\left(I_{3} \times C_{4}\right)$ & 22.44 & 533.90 & 2.92 \\
\hline S.Em \pm & 1.23 & 34.44 & 0.25 \\
\hline C.D. at $5 \%$ & NS & 102.33 & 0.75 \\
\hline Rainfed pigeonpea & 21.32 & 455.6 & - \\
\hline S.Em \pm & 1.21 & 34.56 & - \\
\hline C.D. at $5 \%$ & NS & 100.89 & - \\
\hline
\end{tabular}


Table.3 Grain yield and economics of pigeonpea as influenced by irrigation levels and cropping systems under drip irrigation

\begin{tabular}{|c|c|c|c|c|}
\hline Treatments & $\begin{array}{c}\text { Grain } \\
\text { yield } \\
\left(\mathrm{kg} \mathrm{ha}^{-1}\right)\end{array}$ & $\begin{array}{c}\text { Gross } \\
\text { returns } \\
\left(\text { Rs. ha }^{-1}\right)\end{array}$ & $\begin{array}{c}\text { Net } \\
\text { returns } \\
\left(\text { Rs. ha }^{-1}\right)\end{array}$ & $\begin{array}{c}\mathrm{BC} \\
\text { ratio }\end{array}$ \\
\hline \multicolumn{5}{|l|}{ Main plots: Irrigation levels (I) } \\
\hline $50 \%$ CPE $\left(\mathbf{I}_{1}\right)$ & 2276 & 196020 & 143710 & 3.77 \\
\hline $75 \%$ CPE $\left(I_{2}\right)$ & 2524 & 226245 & 173435 & 4.29 \\
\hline $100 \%$ CPE $\left(\mathbf{I}_{3}\right)$ & 2462 & 218010 & 164700 & 4.10 \\
\hline S.Em \pm & 37 & 4630 & 4630 & 0.08 \\
\hline C.D. at $5 \%$ & 146 & 18210 & 18210 & 0.30 \\
\hline \multicolumn{5}{|l|}{ Sub plot: Cropping systems (C) } \\
\hline Sole pigeonpea $\left(C_{1}\right)$ & 2656 & 159380 & 120820 & 4.13 \\
\hline Pigeonpea + Greengram $\left(C_{2}\right)$ & 2304 & 252400 & 196590 & 4.52 \\
\hline Pigeonpea + Blackgram $\left(\mathbf{C}_{3}\right)$ & 2358 & 230700 & 172640 & 3.97 \\
\hline Pigeonpea + Soybean $\left(\mathbf{C}_{4}\right)$ & 2366 & 211220 & 152410 & 3.59 \\
\hline S.Em \pm & 58 & 5850 & 5850 & 0.12 \\
\hline C.D. at $5 \%$ & 170 & 17390 & 17390 & 0.40 \\
\hline \multicolumn{5}{|l|}{ Interaction effects $(\mathrm{I} \times \mathrm{C})$} \\
\hline $50 \%$ CPE $\times$ Sole pigeonpea $\left(I_{1} \times C_{1}\right)$ & 2501 & 150060 & 112000 & 3.94 \\
\hline $50 \%$ CPE $\times$ Pigeonpea + Greengram $\left(I_{1} \times C_{2}\right)$ & 2171 & 230280 & 174970 & 4.16 \\
\hline $50 \%$ CPE $\times$ Pigeonpea + Blackgram $\left(I_{1} \times C_{3}\right)$ & 2216 & 208260 & 150700 & 3.62 \\
\hline $50 \%$ CPE $\times$ Pigeonpea + Soybean $\left(I_{1} \times C_{4}\right)$ & 2218 & 195480 & 137170 & 3.35 \\
\hline $75 \%$ CPE $\times$ Sole pigeonpea $\left(I_{2} \times C_{1}\right)$ & 2750 & 165000 & 126440 & 4.28 \\
\hline $75 \%$ CPE $\times$ Pigeonpea + Greengram $\left(I_{2} \times C_{2}\right)$ & 2399 & 267960 & 212150 & 4.80 \\
\hline $75 \%$ CPE $\times$ Pigeonpea + Blackgram $\left(I_{2} \times C_{3}\right)$ & 2449 & 246720 & 188660 & 4.25 \\
\hline $75 \%$ CPE $\times$ Pigeonpea + Soybean $\left(I_{2} \times C_{4}\right)$ & 2498 & 225300 & 166490 & 3.83 \\
\hline $100 \%$ CPE $\times$ Sole pigeonpea $\left(I_{3} \times C_{1}\right)$ & 2718 & 163080 & 124020 & 4.18 \\
\hline $100 \%$ CPE $\times$ Pigeonpea + Greengram $\left(I_{3} \times C_{2}\right)$ & 2343 & 258960 & 202650 & 4.60 \\
\hline $100 \%$ CPE $\times$ Pigeonpea + Blackgram $\left(I_{3} \times C_{3}\right)$ & 2408 & 237120 & 178560 & 4.05 \\
\hline $100 \%$ CPE $\times$ Pigeonpea + Soybean $\left(I_{3} \times C_{4}\right)$ & 2381 & 212880 & 153570 & 3.59 \\
\hline S.Em \pm & 95 & 9930 & 9930 & 0.22 \\
\hline C.D. at $5 \%$ & 280 & 29500 & 29500 & 0.65 \\
\hline Rainfed pigeonpea & 1080 & 66880 & 36518 & 2.20 \\
\hline S.Em \pm & 95.57 & 7886 & 7844 & 0.16 \\
\hline C.D. at $5 \%$ & 278.97 & 23018 & 22896 & 0.47 \\
\hline
\end{tabular}

Kumbhar et al., (2015) reported that irrigation scheduling at $0.8 \mathrm{IW} / \mathrm{CPE}$ ratio recorded significantly higher net returns of Rs.37, 591 ha $^{-1}$ with maximum $\mathrm{BC}$ ratio of 2.34 in blackgram. Similar results were reported by Pramod et al., (2006) and Muniyappa et al., (2017) in chickpea and Deewan et al., (2017) in clusterbean. 
Among all the cropping systems, pigeonpea intercropped with greengram in 1:3 row proportions recorded significantly higher gross returns (Rs. 2,52,400 ha ${ }^{-1}$ ), net returns (Rs. 1,96,590 ha $\mathrm{ha}^{-1}$ ) and $\mathrm{BC}$ ratio (4.52) as compared to other intercropping systems. However, significantly lower gross returns (Rs. 1,59,380 $\mathrm{ha}^{-1}$ ) and net returns (Rs. $1,20,820 \mathrm{ha}^{-1}$ ) were recorded in sole pigeonpea but $\mathrm{BC}$ ratio (3.59) in pigeonpea + soybean (1:3). Kantwa et al., (2005) reported that pigeonpea + greengram intercropping system fetched higher gross returns, net return as well as BC ratio than sole pigeonpea. This might be due to marginal difference in yield of pigeonpea and additional yield of greengram, which resulted in higher net return in pigeonpea + greengram cropping systems than in sole pigeonpea.

The interaction effect between scheduling of irrigation and cropping systems on economics was found significant. Scheduling of irrigation at $75 \% \mathrm{CPE}$ with pigeonpea + greengram (1:3) intercropping system recorded significantly higher gross returns (Rs. 2,67,960 ha ${ }^{-1}$ ), net returns (Rs. 2,12,150 $\mathrm{ha}^{-1}$ ) and $\mathrm{BC}$ ratio (4.80) as compared to all other treatment combinations. Significantly lower gross returns (Rs. 1,50,060 ha-1) and net returns (Rs. 1,12,000 $\mathrm{ha}^{-1}$ ) were registered in $50 \%$ CPE with sole pigeonpea but BC ratio(3.35) was recorded in 50\% CPE with pigeonpea + soybean (1:3) intercropping system.

The rainfed pigeonpea recorded significantly lower gross returns (Rs. 66,880 ha ${ }^{-1}$ ), net returns (Rs. 36,518 $\mathrm{ha}^{-1}$ ) and BC ratio (2.20).

It is concluded that among the treatments, 75 $\%$ CPE with sole pigeonpea recorded higher nutrient uptake, protein yield and grain yield but scheduling of irrigation at $75 \% \mathrm{CPE}$ in pigeonpea + greengram (1:3) intercropping system was found to be more productive and profitable system as it has recorded significantly higher net returns and benefit cost ratio when compared to other irrigation scheduling and intercropping systems.

\section{References}

Abraham, T., Thenua, O. V. S. and Shivakumar, B. G., 2010, Impact of levels of irrigation and fertility gradients on dry matter production, nutrient uptake and yield of chickpea (Cicer arietinum) intercropping system. Legume Res., 33(1): 10-16.

Al-Kaisi, M. M. and Yin, X., 2003, Effects of nitrogen rate, irrigation rate and plant population on corn (Zea mays L.) yield and water use efficiency. Agron. J., 95 : 1475-1482.

Anonymous, 2018, Directorate of economics and statistic.www.indianstat.com

Ansari, M. A., Rana, K. S., Rana, D. S., Kumar, A. and Hariom, P. H. D., 2012, Effect of anti-transpirant and nutrient management on pearl millet (Pennisetum glaucum) and pigeonpea (Cajanus cajan) intercropping system under rainfed conditions. Indian $J$. Agron., 57: 343-348.

Chandra sekhar, P., Avil kumar, K., Uma devi, M. and Ramulu, V., 2015, Nutrient uptake of rabi pigeon pea (Cajanus cajan (L) Millsp) as influenced by different nutrigation levels. The J. Res. PJTSAU, 43(3): 41 45.

Deewan, P., Regar, K. L., Jajoriya, M., Meena, M. and Verma, R., 2017, Influence of irrigation scheduling (IW/CPE ratios) and plant growth regulators on quality, yield and economics of summer clusterbean (Cyamopsis tetragonoloba L.) under middle Gujarat conditions. Int. J. Chem. Stud., 5(5): 467-471.

Hebbar, S. S., Ramachandrappa, B. K., Nanjappa, H. V. and Prabhakar, M., 2004, Studies on NPK drip fertigation in field grown tomato (Lycopersicon esculentum Mill.). European J. Agron., 21: 117-127.

Jadhav, K. T., Chavan, A. S., Raskar, S. K. and Lahase, R. U., 2018, Influence of spacing and drip irrigation on yield attributes, productivity and economics of pigeonpea 
(Cajanus cajan L.). Int. J. Curr. Microbiol. App. Sci., 7(2): 3498-3506.

Kantwa, S. R., Ahlawat, I. P. S. and Gangaiah, B., 2005, Performance of sole and intercropped pigeonpea (Cajanus cajan L.) as influenced by land configuration, postmonsoon irrigation and phosphorus fertilization. Indian J. Agric. Sci., 76(10): 635-637.

Kiran, R., 2014, Effect of methods and scheduling of irrigation on growth, yield attributes and yield of pigeonpea [Cajanus cajan (L.) millsp]. Ph.D. Thesis., College of Agric. Gwalior (M.P.), Rajmata Vijayaraje Scindia Krishi Vishwa Vidyalaya

Kumbhar, N. M., Patel, J. S., Gediya, K. M., Suryawanshi and Patel, C. J., 2015, Influence of irrigation scheduling (IW/CPE) and sulphur on yield of blackgram (Vigna mungo L.). Madras Agric. J., 95(1/6): 57-60.

Mahalakshmi, K., Avil, K. K., Reddy, M. D. and Uma, D. M., 2011, Response of rabi pigeonpea [Cajanus cajan (L.)] to different levels of drip irrigation. J. Res. ANGRAU, 39(4): 101-103.

Mansur, C. P., Palled, Y. B., Halikatti, S. I., Chetti, M. B. and Salimath, P. M., 2010, The effect of dates of sowing and irrigation levels on growth and yield of chickpea (Cicer arietinum L.). Karnataka J. Agric. Sci., 23(3): 461-463.

Muniyappa, Mudalagiriyappa, Ramachandrappa, B. K., Nagaraju and Sathish, A., 2017, Studies on the influence of depth and interval of drip irrigation on yield, water use efficiency and economics of chickpea (Cicer arietinum L.). Int. J. Pure App. Biosci., 5(1): 771-776.

Patil, A.B. and Padmani, D.R., 2007, Nutrient uptake pattern of pigeonpea (Cajanus cajan) as influenced by integrated nutrient management. Internat. J. Agric. Sci., 3(2): 176-178.

Pramod, M. C., Arun, B. K., Pankaj, U. R. and Sanjay, S. C., 2006, Effect of intercropping of pigeonpea, sorghum and cotton on productivity and yield advantages of soybean (Glycine max L.) under rainfed condition. Int. J. Agric. Sci., 2(2): 478-479.

Ranjitha, P. S., Ramulu, V., Jayasree, G. and Reddy, S. N., 2018, Growth, Yield and Water Use Efficiency of Groundnut (Arachis hypogaea) under Drip and Surface Furrow Irrigation. Int. J. Curr. Microbiol. App. Sci., 7(9): 1371-1376.

Shirgapure, K. H. and Fathima, P. S., 2018, Growth and yield of pulses as influenced by irrigation levels in southern dry zone of Karnataka. J. Pharmacogn. Phytochem., 7(1): 2444-2448

Singh, J., Supriya, Saxena, S., Mishra, P. and Singh, R. B., 2018, Effect of fertigation doses and amount of water applied on the growth and yield of pigeonpea (Cajanus Cajan) CV. PUSA 992. J. Pharmacogn. Phytochem., 2: 182-185.

Thanki, J. D. and Solanki, R. K., 2010, Response of rabi pigeonpea (Cajanus Cajan) to moisture regimes and fertilizer management under South Gujarat condition. Green Farming, 1(3): 257-259.

\section{How to cite this article:}

Megharani, Pandit, S. Rathod, B. M. Dodamani, Devappagouda H. Patil and Patil, R. P. 2020. Effect of Irrigation Levels and Cropping Systems on Quality Parameters and Economics of Pigeonpea. Int.J.Curr.Microbiol.App.Sci. 9(02): 2244-2253.

doi: https://doi.org/10.20546/ijcmas.2020.902.254 